\title{
Incidence of Readmission in Patients in Sulaimani Emergency Hospital
}

\author{
Faruk H Faraj (MBChB, FRCS, FICS, CABS) ${ }^{1}$ and Authman Y Ali \\ $(\mathrm{MBChB}, \mathrm{HD}, \mathrm{A} \& \mathrm{E}, \text { Surgery })^{2}$
}

\begin{abstract}
Background:Readmission after hospitalization is a preventable experience which can impose huge costs on the patients and the health care systems. Studies have reported that readmission of patients within 30 days following discharge is quite common and associated with some clinical and nonclinical factors. Mental and behavioral health issues, alcoholrelated disorders, pregnancy, diabetes, schizophrenia, and mood disorders have been reported as the commonest conditions that can cause readmission. Research has also stated that patient readmission can show low quality of health care services.

Objective: To assess the incidence of patients who had been readmitted to emergency room in Sulaimani Emergency Hospital during three consecutive months.

Patients and Methods:The present prospective study which was carried out on 126 readmitted patients who were selected from 3264 patients who had referred to Sulaimani Accident \& Emergency Hospital in Kurdistan Region of Iraq from (1st. Jun 2018 ) to ( 31st.Aug. 2018 ) . A researcher-designed checklist was used to collect the required data. The collected data were analyzed through descriptive statistics using Statistical Package for Social Sciences version 22.0, and the results were presented as frequencies and percentages in tables.

Results: The results of the present study revealed that the hospital readmission rate was lower among the patients older than 50 years. Also, female patients accounted for a larger population of readmitted patients than the males. There was no significant association found between gender and readmission. Epigastric and abdominal pains were the most frequent symptoms and presentations observed at readmission. In addition, associated medical diseases and surgical procedures were found to have outstanding effect on readmission. The most common detected disorders among the readmitted patients were suspected acute appendicitis and acute pancreatitis. In addition the type of medical intervention was found to have a remarkable effect on the rate of readmission. A significant relationship was observed between the observed clinical presentations and the readmitted patients' age, occupation, and marital status. Also, the readmitted patients' past surgical history and their age, gender, occupation, and marital status were significantly correlated.

Conclusion: Hospital readmission was found to be common among patients who had associated medical diseases, surgical procedures, suspected acute appendicitis, and acute pancreatitis; therefore, such patients need to be provided with more careful care and proper
\end{abstract}


medical interventions in order to prevent or reduce readmission rate and consequently cut costs. Moreover, while admitting and readmitting patients, their age, gender, marital status, and occupation need to be taken into close account because these variables are effective in their clinical presentation and correlated with their past surgical history.

Keywords: Readmission, Hospitalization, Emergency room, The Kurdistan Region of Iraq. Corresponding Author: authmanaldulaimi@gmail.com

Received: $11^{\text {th }}$ July 2019

Accepted: $19^{\text {th }}$ August 2019

DOI:https://doi.org/10.26505/DJM.17024780711

\footnotetext{
${ }^{1}$ Consultant Surgeon in Sulaimani Teaching Hospital- Head master of Iraqi Board for General Surgery- Sulaimani center -Iraq.

${ }^{2}$ Ministry of Health- Baghdad alkerkh-Iraq
}

\section{Introduction}

Being readmitted after hospitalization is an often preventable event which can be quite costly. According to a study conducted by Hansen et al 2, about one-fifth of Medicare beneficiaries - over 2.3 million patients were re-hospitalized within 30 days of discharge during 2003 and 2004 [11]. The costs imposed on Medicare as a result of readmission during 30 days has been estimated over $\$ 17$ billion dollars annually ${ }^{2}$. A 2006 Commonwealth Fund report estimated that Medicare would save \$1.9 billion annually if national readmission rates were lowered to the levels achieved by the top-performing regions. In 2009, the Centers for Medicare and Medicaid Services began reporting hospital readmission rates to the public and penalizing hospitals by reducing payments for readmissions within 30 days. It is estimated that nearly $20 \%$ of all Medicare beneficiaries are readmitted within 30 days, costing the Centers for Medicare and Medicaid Services approximately 17.4 billion USD annually and leading to increased mortality [3].
However, readmission of general medicine patients within 30 days is quite common and associated with some simply detectable clinical and nonclinical factors. Identifying these risk factors can help health care providers control the interventions in a way to reduce potentially avoidable readmissions, which useful to focus resources to optimize the discharge process and reduce avoidable readmissions[4,5]. Medicaid readmissions can be resulted from different conditions, with most relating to mental and behavioral health issues. Alcohol-related disorders, pregnancy, diabetes mellitus, schizophrenia and other psychotic disorders, and mood disorders are among the top conditions leading to readmissions. Chemotherapy or radiation maintenance topped the list of conditions leading to privately insured readmissions, followed by septicemia, device, implant, or graft complications, surgical complications, and mood disorders. Identifying patients at risk for these conditions is a good place to start. Data analysis and risk stratification tools are 
crucial to identifying patients who are the most likely to get readmitted in the hospital following discharge[6,7].Risk factors for readmission have been reported as poor social support, functional disability, multiple co morbidities, myocardial infarction, chronic kidney disease, chronic obstructive pulmonary disease, pneumonia, and congestive heart failure (CHF) [8] . Patients with dementia experience a 3 -fold increase of preventable readmissions and need to be provided with more healthcare services than those without dementia. Currently, the Centers for Medicaid Services (CMS) and Medicare publicly report risk-standardized readmission rates for acute myocardial infarction, pneumonia, and heart failure [9] . Hospital readmission measures have also been developed by CMS for stroke and for hip and knee replacement. It has also developed such measures for vascular procedures and chronic obstructive pulmonary diseases. While it is helpful to assess readmission rates for specific groups of patients, these conditions account only for a small portion of total readmissions. By contrast, a hospital-wide, all-condition readmission measure is needed to provide a broader assessment of the quality of care at hospitals [7]. Given the significance of the issue of readmission and taking into account the cost it imposes on the patients and health care systems globally, the present study was carried out in order to examine the incidence of patients re-admitted to Accident \& Emergency (A. \& E.) Hospital of Sulaimani during three consecutive months.

\section{Patients and Methods}

In the present prospective study, ethical considerations were taken into account by obtaining necessary approvals from the Scientific and Ethical Committee of Sulaimani University / College of Medicine. The study was a cross-sectional descriptive investigation which was conducted during 3 months from $\left(1^{\text {st }}\right.$.June 2018$)$ to $\left(31^{\text {st }}\right.$.Aug. 2018). The study sample consisted of 126 readmitted patients who were selected from 3264 patients who had referred to Sulaimani Surgical Emergency Hospital.In order to collect required data, a researcher-designed checklist was utilized to gather the data from those 126 readmitted patients' files. For this purpose, the medical community department of the hospital assisted the researcher. The collected data included the patients' information about their demographics (i.e. age, gender, occupation, place of residence, and marital status) and their medical records (i.e. duration, presentations at the last admission , past surgical history, lab investigations, imaging studies, Professional diagnosis, previous examinations, cause of discharging, and previous interventions).

\section{The inclusion criteria are:}

- Age $\geq 10$ years

- Both genders

- Readmission after being discharged

- Having complete medical records in their hospital file

The exclusion criteria are:

-Being discharged without readmission -Incomplete information in their hospital file 


\section{Statistical analysis}

The collected data were analyzed through SPSS (Statistical Package for Social Sciences) version 22.0. For this purpose, descriptive statistics was employed, and the results were presented as frequencies and percentages in tables.

\section{Results}

Regarding the biographic aspects of the patients: The most common age groups were between 2 nd -4 th decades of life accounting for over half of the patients with females were slightly more and over $1 / 3$ rd of them were self-employed as shown in Table(1)

Table (1): Shows the biographic aspects of the readmitted patients

\begin{tabular}{|c|c|c|}
\hline Variables & Frequency & Percentage (\%) \\
\hline \hline Age & & \\
$10-20$ & 35 & 27.8 \\
$21-30$ & 36 & 28.6 \\
$31-40$ & 24 & 19.0 \\
$41-50$ & 13 & 10.3 \\
$>50$ & 18 & 14.3 \\
Total & 126 & 100.0 \\
\hline \hline Gender & & 50.7 \\
\hline Female & 64 & 49.3 \\
Male & 62 & \\
\hline Occupation & & 30.2 \\
\hline Self-employed & 38 & 29.4 \\
Housewife & 37 & 24.6 \\
Pupil or student & 31 & 11.1 \\
Fixed job & 14 & 4.8 \\
Unemployed & 6 & 100.0 \\
Total & 126 & \\
\hline Marital status & & 51.6 \\
\hline Married & 65 & 37.3 \\
Single & 47 & 9.5 \\
Widowed & 12 & 1.6 \\
Divorced & 2 & 100.0 \\
Total & 126 & \\
\hline \multicolumn{2}{|c|}{} \\
\hline
\end{tabular}

The most common presentation in the readmitted cases was right lower abdominal pain found in 62 patients $(49.2 \%)$, while epigastric pain was the most common presentation in the previous admission as shown in Table(2). 
Table (2): Shows the Frequency \& percentage of the presentations in the readmitted $\& 1^{\text {st }}$ admission

\begin{tabular}{||l||c|c||}
\hline \multicolumn{1}{|c||}{ Presentation } & Frequency $\mathbf{( N )}$ & $\begin{array}{c}\text { Percentage } \\
(\mathbf{\%})\end{array}$ \\
\hline \hline Readmitted patients & & \\
Right lower abdominal pain & 62 & 49.2 \\
Epigastric pain & 27 & 21.4 \\
Nonspecific abdominal pain & 9 & 7.1 \\
RIF pain & 8 & 6.3 \\
Abdominal pain and dysuria & 6 & 4.8 \\
Right upper abdominal pain & 4 & 3.2 \\
Abdominal pain and constipation & 3 & 2.4 \\
Central abdominal pain & 3 & 2.4 \\
Right hypochondrium pain & 2 & 1.6 \\
General abdominal pain & 1 & 0.8 \\
Vomiting & 1 & 0.8 \\
Total & 126 & 100.0 \\
& & \\
\hline Presentation of the previous & & \\
admission & & 33.3 \\
Epigastric pain and dyspepsia & 42 & 26.2 \\
Lower abdominal pain & 33 & 15.1 \\
Nonspecific abdominal pain & 19 & 7.9 \\
Central abdominal pain & 10 & 7.1 \\
Abdominal pain with vomiting & 9 & 4.8 \\
Upper abdominal pain and & 6 & 4.0 \\
dyspepsia & 4 & 2.4 \\
Vague abdominal pain & 3 & \\
Abdominal pain with mild dysuria & & \\
\hline
\end{tabular}

The hospital stay varied between 1-36 days

with mean hospital stay 4.5 days as shown in

Table (3).

Table (3): Show duration of hospitalization of the readmitted patients

\begin{tabular}{|c||c||c|}
\hline Duration (day) & Frequency (N) & Percentage (\%) \\
\hline $\mathbf{1}$ & 17 & 13.5 \\
\hline $\mathbf{2}$ & 31 & 24.6 \\
\hline $\mathbf{3}$ & 18 & 14.3 \\
\hline \hline $\mathbf{4}$ & 11 & 8.7 \\
\hline \hline $\mathbf{5}$ & 17 & 13.5 \\
\hline \hline $\mathbf{6}$ & 9 & 7.1 \\
\hline \hline $\mathbf{7}$ & 11 & 8.7 \\
\hline \hline $\mathbf{9}$ & 3 & 2.4 \\
\hline \hline $\mathbf{1 8}$ & 2 & 1.6 \\
\hline \hline $\mathbf{2 0}$ & 1 & 0.8 \\
\hline \hline $\mathbf{3 6}$ & 5 & 4.0 \\
\hline \hline Total & 1 & 0.8 \\
\hline \hline
\end{tabular}


In Regarding past surgical history (PSH), the results showed that appendectomy were observed in 14 patients (11.1), left hemorrhagic ovarian cystectomy in 1 patient $(0.8 \%)$, right breast mass excision in 1 patient $(0.8 \%)$, tonsillectomy in 2 patients
$(1.6 \%)$, cholecystectomy in 7 patients (5.6\%), and appendectomy with cholecystectomy in 1 patient $(0.8 \%)$. Moreover, 100 patients $(79.4 \%)$ did not have any PSH Table (4).

Table (4): Frequency of past surgical history in the patients

\begin{tabular}{|l||c||c|}
\hline \multicolumn{1}{|c|}{ PSH } & Frequency $(\mathbf{N})$ & Percentage (\%) \\
\hline \hline Appendectomy & 14 & 11.1 \\
\hline \hline Cholecystectomy & 7 & 5.6 \\
\hline Tonsillectomy & 2 & 1.6 \\
\hline Left hemorrhagic ovarian cystectomy & 1 & 0.8 \\
\hline Rt. breast mass excision & 1 & 0.8 \\
\hline \hline Appendectomy \& Cholecystectomy & 1 & 0.8 \\
\hline \hline No PSH. & 100 & 79.4 \\
\hline \hline Total & 126 & 100.0 \\
\hline
\end{tabular}

As show in Table (5), CBC was carried out for all of the patients $(100 \%)$, GUE for 81 patients $(64.3 \%)$, RBS for 79 patients (62.7\%), LFTS and RFT for 42 patients $(33.3 \%)$, S. amylase for 36 patients $(28.6 \%)$,
S. lipase for 35 patients $(27.8 \%)$, lipid profile for 17 patients $(13.5 \%)$, B. urea for 13 patients (10.3\%), ECG for 4 patients (3.2\%), and HbA1c and CRP for 1 patient $(0.8 \%)$ Table (5).

Table (5): Show the frequency \& Percentage of the lab. Investigations carried out for the patients in this

\begin{tabular}{|c|c|c|}
\hline Lab investigations & Frequency $(\mathbf{N})$ & Percentage (\%) \\
\hline \multicolumn{3}{|l|}{ CBC } \\
\hline Yes & 126 & 100.0 \\
\hline \multicolumn{3}{|l|}{ S. Amylase } \\
\hline Yes & 36 & 28.6 \\
\hline \multicolumn{3}{|l|}{ S. Lipase } \\
\hline Yes & 35 & 27.8 \\
\hline \multicolumn{3}{|l|}{ Lipid profile } \\
\hline Yes & 17 & 13.5 \\
\hline \multicolumn{3}{|l|}{ B. urea } \\
\hline Yes & 13 & 10.3 \\
\hline \multicolumn{3}{|l|}{ RBS } \\
\hline Yes & 79 & 62.7 \\
\hline \multicolumn{3}{|l|}{ HbA1c } \\
\hline Yes & 1 & 8 \\
\hline \multicolumn{3}{|l|}{ Abd. x-ray ( KUB ) } \\
\hline Yes & 1 & 0.8 \\
\hline
\end{tabular}




\begin{tabular}{|c|c|c|}
\hline Yes & 42 & 33.3 \\
\hline \multicolumn{3}{|l|}{ RFT } \\
\hline Yes & 42 & 33.3 \\
\hline \multicolumn{3}{|l|}{ ECG } \\
\hline Yes & 4 & 3.2 \\
\hline \multicolumn{3}{|l|}{ CRP } \\
\hline Yes & 1 & 0.8 \\
\hline \multicolumn{3}{|l|}{ GUE } \\
\hline Yes & 81 & 64.3 \\
\hline
\end{tabular}

Abdominal U/S performed for 46 patients patients (13.5\%) ECG, and 4 (3.2\%) (36.5\%), 30 (23.8\%) abdominal X-ray, 17 abdominal CT scan See Table (6).

Table (6): Show the imaging techniques employed for the patients

\begin{tabular}{|c|c|c|}
\hline Imaging & Frequency (N) & Percentage (\%) \\
\hline \multicolumn{3}{|l|}{ Abdominal U/S } \\
\hline Yes & 46 & 36.5 \\
\hline \multicolumn{3}{|l|}{ Abdominal X-ray } \\
\hline Yes & 30 & 23.8 \\
\hline \multicolumn{3}{|l|}{ Abdominal CT scan } \\
\hline 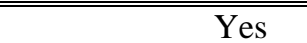 & 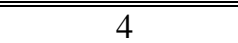 & 3.2 \\
\hline \multicolumn{3}{|l|}{ ECG } \\
\hline 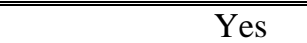 & $\overline{177}$ & 13.5 \\
\hline
\end{tabular}

Regarding the diagnosis results, it was observed that 6 patients $(4.8 \%)$ had acute cholecystitis, $4(3.2 \%)$ had acute gastritis, 16 $(12.7 \%)$ acute pancreatitis, $1(0.8 \%)$ acute abdominal pain, $2(1.6 \%)$ appendicitis with Rt. ovarian cyst, $15(11.9 \%)$ acute appendicitis, 8 (6.3\%) UTI, and 47 (37.3\%) suspected acute appendicitis Table (7).

Table (7): Show the diagnosis results

\begin{tabular}{||l||c||c|}
\hline \multicolumn{1}{|c||}{ Diagnosis } & Frequency (N) & Percentage (\%) \\
\hline \hline Acute cholecystitis & 6 & 5.9 \\
\hline \hline Acute gastritis & 4 & 3.9 \\
\hline \hline Acute pancreatitis & 16 & 15.8 \\
\hline Acute abdominal pain & 1 & 0.9 \\
\hline \hline Appendicitis with Rt. ovarian cyst & 2 & 1.9 \\
\hline \hline Acute Appendicitis & 15 & 14.8 \\
\hline \hline Perforated DU First part & 2 & 1.9 \\
\hline UTI & 8 & 7.9 \\
\hline \hline Suspected acute appendicitis & 47 & 46.1 \\
\hline \hline \multicolumn{1}{|c||}{ Total } & 103 & 99.1 \\
\hline
\end{tabular}

The results of analyzing the first admission received by the patients indicated that most of

them (96\%) had received CBC, followed by 80 patients (63.5\%) had GUE, 71 (56.3\%) RBS, 43 
(34.1\%) abdominal U/S, 41 (32.5\%) S. amylase, $33(26.2 \%)$ RFT, 30 (23.8\%) LFT, $29(23 \%)$ abdominal X-ray, 26 (20.6\%) US, 17 (13.5\%)
ECG, $14(11.1 \%)$ B. urea, 7 (5.6\%) S. creatinine, $6(4.8 \%)$ S. electrolytes, 3 (2.4\%) lipid profile, 1 $(0.8 \%)$ HbAlc, and $1(0.8 \%)$ GSE Table (8).

Table (8): Show frequency \& Percentage of investigations of patients in $1^{\text {st. }}$ admission

\begin{tabular}{|c|c|c|}
\hline Previous investigations if present & Frequency $(\mathbf{N})$ & Percentage (\%) \\
\hline \multicolumn{3}{|l|}{ ECG } \\
\hline Yes & 17 & 13.5 \\
\hline \multicolumn{3}{|l|}{ CBC } \\
\hline Yes & 121 & 96.0 \\
\hline \multicolumn{3}{|l|}{ GUE } \\
\hline Yes & 80 & 63.5 \\
\hline \multicolumn{3}{|l|}{ RBS } \\
\hline Yes & 71 & 56.3 \\
\hline \multicolumn{3}{|l|}{ S. Amylase } \\
\hline Yes & 41 & 32.5 \\
\hline \multicolumn{3}{|l|}{ Lipid profile } \\
\hline Yes & 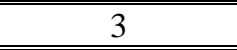 & 2.4 \\
\hline \multicolumn{3}{|l|}{ LFT } \\
\hline Yes & 30 & 23.8 \\
\hline \multicolumn{3}{|l|}{ S.Electrolytes } \\
\hline Yes & 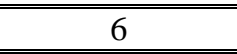 & 4.8 \\
\hline \multicolumn{3}{|l|}{ B .urea } \\
\hline Yes & 14 & 11.1 \\
\hline \multicolumn{3}{|l|}{ S. Creatinine } \\
\hline Yes & 7 & 5.6 \\
\hline \multicolumn{3}{|l|}{ Abdominal U/S } \\
\hline Yes & 43 & 34.1 \\
\hline \multicolumn{3}{|l|}{ HbA1c } \\
\hline Yes & 1 & 0.8 \\
\hline \multicolumn{3}{|l|}{ GSE } \\
\hline Yes & 1 & 0.8 \\
\hline \multicolumn{3}{|l|}{$\mathrm{U} / \mathrm{S}$} \\
\hline Yes & 26 & 20.6 \\
\hline \multicolumn{3}{|l|}{ RFT } \\
\hline Yes & 33 & 26.2 \\
\hline \multicolumn{3}{|l|}{ Abdominal X-ray } \\
\hline Yes & 29 & 23.0 \\
\hline
\end{tabular}

The results of the present study regarding the causes of the patients' discharge showed that most of them $(73 \%)$ were discharged due to refined management and pain relief,
18 patients $(14.3 \%)$ were discharged on their responsibility, $6(4.8 \%)$ due to improvement and discharge, and $1(0.8 \%)$ due to refined operation Table (9). 
Table (9): Cause of discharging the readmitted patients

\begin{tabular}{||l|c|c||}
\hline \multicolumn{1}{|c|}{ Cause of discharge } & Frequency (N) & Percentage (\%) \\
\hline \hline Discharge on their responsibility & 18 & 14.3 \\
\hline \hline Improvement and discharge & 6 & 4.8 \\
\hline \hline Refined management and Pain relief & 92 & 73.0 \\
\hline \hline Refined operation & 1 & 0.8 \\
\hline
\end{tabular}

With regard to the previous intervention the patients had received, the results of the present study showed that most of them $(87.3 \%)$ had conservative management, 9 patients $(7.1 \%)$ had been prepared for appendectomy, and 2 patients (1.6\%) had been suspected acute appendicitis and need further evaluation Table (10).

Table (10): Previous interventions received by the patients

\begin{tabular}{|c|c|c|}
\hline Previous intervention & Frequency $(\mathbf{N})$ & Percentage (\%) \\
\hline \multicolumn{3}{|l|}{ Prepared for appendectomy } \\
\hline No & 117 & 92.9 \\
\hline Yes & 9 & 7.1 \\
\hline \multicolumn{3}{|l|}{ Suspected acute appendicitis } \\
\hline No & 124 & 98.4 \\
\hline Yes & 2 & 1.6 \\
\hline \multicolumn{3}{|l|}{ Conservative management } \\
\hline No & 16 & 12.7 \\
\hline Yes & 110 & 87.3 \\
\hline
\end{tabular}

With regard to the association between the patients' age and the presentations observed during their readmission, the results of the present study indicated that these two variables were significantly correlated $(\mathrm{p}=0.002)$, such that most of the patients aged 10-20, 21-30, and 31-40 had right lower abdominal pain, while those who were 41-50 and more had almost all of the representations Table(11). 
Table (11): The association between the patients' age and their presentations at readmission

\begin{tabular}{|c|c|c|c|c|c|c|c|c|}
\hline & \multicolumn{5}{|c|}{ Age } & \multirow{2}{*}{ Total } & \multirow{2}{*}{$\begin{array}{c}\mathrm{P}- \\
\text { value }\end{array}$} \\
\hline & & 10-20 & 21-30 & 31-40 & 41-50 & $>50$ & & \\
\hline \multirow{11}{*}{ 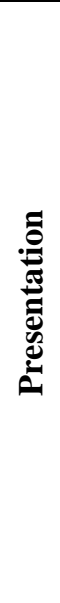 } & Non-specific abd. pain & $3(33.3)$ & $3(33.3)$ & $0(0.0)$ & $2(22.2)$ & $\overline{~ 1(11.1)}$ & $9(100.0)$ & \multirow{12}{*}{0.002} \\
\hline & $\begin{array}{l}\text { General abd. pain } \\
\end{array}$ & $0(0.0)$ & $0(0.0)$ & $0(0.0)$ & $0(0.0)$ & $1(100.0)$ & 1 1(100.0) & \\
\hline & RIF pain & $4(50.0)$ & $3(37.5)$ & $1(12.5)$ & $0(0.0)$ & "0(0.0) & $8(100.0)$ & \\
\hline & $\begin{array}{c}\text { Rt. hypochondrium } \\
\text { pain }\end{array}$ & $0(0.0)$ & $0(0.0)$ & $1(50.0)$ & $1(50.0)$ & $0(0.0)$ & $2(100.0)$ & \\
\hline & $\begin{array}{l}\text { Abd. pain \& } \\
\text { constipation }\end{array}$ & $1(33.3)$ & $1(33.3)$ & $0(0.0)$ & $0(0.0)$ & $1(33.3)$ & $3(100.0)$ & \\
\hline & Abd. pain \& dysuria & $0(0.0)$ & $1(16.7)$ & $1(16.7)$ & $2(33.3)$ & $2(33.3)$ & $6(100.0)$ & \\
\hline & Central Abd. pain & $1(33.3)$ & $1(33.3)$ & $1(33.3)$ & $0(0.0)$ & $0(0.0)$ & $3(100.0)$ & \\
\hline & Vomiting & $0(0.0)$ & $1(100.0)$ & $0(0.0)$ & $0(0.0)$ & $0(0.0)$ & $1(100.0)$ & \\
\hline & Rt. upper Abd. pain & $0(0.0)$ & $0(0.0)$ & $2(50.0)$ & $2(50.0)$ & $0(0.0)$ & 4 4(100.0) & \\
\hline & Epigastric pain & $2(7.4)$ & $3(11.1)$ & $8(29.6)$ & $4(14.8)$ & $10(37.0)$ & $27(100.0)$ & \\
\hline & Rt. lower Abd. pain & $24(38.7)$ & $23(37.1)$ & $\overline{10(16.1)}$ & $2(3.2)$ & $3(4.8)$ & 62(100.0) & \\
\hline & Total & $35(27.8)$ & $36(28.6)$ & $24(19.0)$ & 13(10.3) & $18(14.3)$ & $126(100.0)$ & \\
\hline
\end{tabular}

Moreover, the results of the present study revealed that there was a significant relationship between the patients of occupation and their representation at readmission $(\mathrm{p}=0.01)$. It was seen that right lower abdominal pain was the most frequent presentation at readmission among all occupation types Table (12).

Table (12): The association between the patients' occupation and their presentations at readmission

\begin{tabular}{|c|c|c|c|c|c|c|c|c|}
\hline & \multicolumn{5}{|c|}{ Occupation } & \multirow[b]{2}{*}{ Total } & \multirow{2}{*}{$\begin{array}{c}\text { P- } \\
\text { value }\end{array}$} \\
\hline & & $\begin{array}{l}\text { Pupil \& } \\
\text { student }\end{array}$ & $\begin{array}{c}\text { Fixed } \\
\text { job }\end{array}$ & $\begin{array}{c}\text { Self- } \\
\text { employed }\end{array}$ & Unemployed & Housewife & & \\
\hline \multirow{12}{*}{ 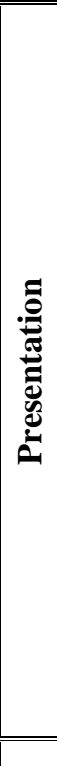 } & $\begin{array}{c}\text { Nonspecific } \\
\text { abd.pain }\end{array}$ & $1(11.1)$ & $1(11.1)$ & $5(55.6)$ & $0(0.0)$ & $2(22.2)$ & $9(100.0)$ & \multirow{12}{*}{0.01} \\
\hline & General abd. pain & $0(0.0)$ & $0(0.0)$ & $0(0.0)$ & $\bar{~} 0(0.0)$ & 10 & 10 & \\
\hline & RIF pain & $1(12.5)$ & $0(0.0)$ & $3(37.5)$ & $0(0.0)$ & $4(50.0)$ & $8(100.0)$ & \\
\hline & $\begin{array}{c}\text { Rt. } \\
\begin{array}{c}\text { Hypochondrium } \\
\text { pain }\end{array} \\
\end{array}$ & $0(0.0)$ & $0(0.0)$ & $2(100.0)$ & $0(0.0)$ & $0(0.0)$ & $2(100.0)$ & \\
\hline & $\begin{array}{l}\text { Abd pain \& } \\
\text { constipation }\end{array}$ & 1(33.3) & $1(33.3)$ & $0(0.0)$ & $0(0.0)$ & $1(33.3)$ & $3(100.0)$ & \\
\hline & $\begin{array}{c}\text { Abd pain \& } \\
\text { dysuria }\end{array}$ & $0(0.0)$ & $0(0.0)$ & $2(33.3)$ & $2(33.3)$ & $2(33.3)$ & $6(100.0)$ & \\
\hline & Central Abd, pain & $1(33.3)$ & 1(33.3) & $21(33.3)$ & $0(0.0)$ & $0(0.0)$ & $3(100.0)$ & \\
\hline & Vomiting & $0(0.0)$ & $0(0.0)$ & $1(100.0)$ & $0(0.0)$ & $0(0.0)$ & $1(100.0)$ & \\
\hline & $\begin{array}{l}\text { Rt. upper Abd. } \\
\text { pain }\end{array}$ & $0(0.0)$ & $0(0.0)$ & $2(50.0)$ & $0(0.0)$ & $2(50.0)$ & $4(100.0)$ & \\
\hline & Epigastric pain & $1(3.7)$ & $2(7.4)$ & $9(33.3)$ & $4(14.8)$ & 11(40.7) & $27(100.0)$ & \\
\hline & Rt. lower Abd. pain & $26(41.9)$ & $9(14.5)$ & $13(21.0)$ & $0(0.0)$ & $14(22.6)$ & $62(100.0)$ & \\
\hline & Total & $31(24.6)$ & $14(11.1)$ & $38(30.2)$ & $6(4.8)$ & $37(29.4)$ & $126(100.0)$ & \\
\hline
\end{tabular}


Between the patients' marital status and their presentations at readmission $(\mathrm{p}=0$. As indicated by the results of the present study, there was a significant association 007). It was observed that right lower abdominal

Table (13): The association between the patients'marital status and their presentations at readmission

\begin{tabular}{|c|c|c|c|c|c|c|c|}
\hline & \multicolumn{4}{|c|}{ Marital status } & \multirow{2}{*}{ Total } & \multirow{2}{*}{ P-value } \\
\hline & & Single & Married & Widowed & Divorced & & \\
\hline \multirow{11}{*}{ 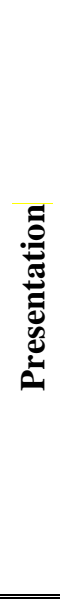 } & Nonspecific abd. pain & $4(44.4)$ & $5(55.6)$ & $0(0.0)$ & $0(0.0)$ & $9(100.0)$ & \multirow{12}{*}{0.007} \\
\hline & General abd. pain & $0(0.0)$ & $0(0.0)$ & $1(100.0)$ & $0(0.0)$ & $1(100.0)$ & \\
\hline & RIF pain & $3(37.5)$ & $5(62.5)$ & $0(0.0)$ & $0(0.0)$ & $8(100.0)$ & \\
\hline & $\begin{array}{l}\text { Rt. hypochondrium } \\
\text { pain }\end{array}$ & $0(0.0)$ & $2(100.0)$ & $0(0.0)$ & $0(0.0)$ & $2(100.0)$ & \\
\hline & $\begin{array}{l}\text { Abd. pain \& } \\
\text { constipation }\end{array}$ & $2(66.7)$ & $1(33.3)$ & $0(0.0)$ & $0(0.0)$ & $3(100.0)$ & \\
\hline & Abd. pain \& dysuria & $101(16.70$ & $3(50.0)$ & $2(33.3)$ & $0(0.0)$ & $6(100.0)$ & \\
\hline & Central Abd, pain & $2(66.7)$ & 1(33.3) & $0(0.0)$ & $0(0.0)$ & $3(100.0)$ & \\
\hline & Vomiting & $1(100.00$ & $0(0.0)$ & $0(0.0)$ & $0(0.0)$ & $1(100.0)$ & \\
\hline & Rt. upper Abd. pain & $0(0.0)$ & 4(100.0) & $0(0.0)$ & $0(0.0)$ & $4(100.0)$ & \\
\hline & Epigastric pain & $4(14.8)$ & 14(51.9) & $7(25.9)$ & $2(7.4)$ & $27(100.0)$ & \\
\hline & Rt. lower Abd. pain & $30(48.4)$ & $30(48.4)$ & $2(3.2)$ & $0(0.0)$ & $62(100.0)$ & \\
\hline & Total & $47(37.3)$ & $65(51.6)$ & $12(9.5)$ & $2(1.6)$ & $126(100.0)$ & \\
\hline
\end{tabular}

In terms of the relationship between the patients' past surgical history (PSH) and their age, the results of the present study demonstrated that there was a significant association between the two variables at

Table (14): The association between the patients' past surgical history and their age

\begin{tabular}{|c|c|c|c|c|c|c|c|c|}
\hline & \multicolumn{5}{|c|}{ Age } & \multirow{2}{*}{ Total } & \multirow{2}{*}{ P-value } \\
\hline & & $1010-20$ & 21-30 & $31-40$ & $41-50$ & $>\mathbf{5 0}$ & & \\
\hline \multirow{5}{*}{ 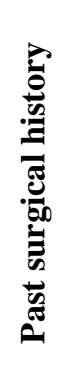 } & Appendectomy & $1(7.1)$ & $2(14.3)$ & $5(35.7)$ & $2(14.3)$ & $4(28.6)$ & $14(100.0)$ & \multirow{5}{*}{0.000} \\
\hline & $\begin{array}{c}\text { Left } \\
\text { hemorrhagic } \\
\text { ovarian } \\
\text { cystectomy }\end{array}$ & $0(0.0)$ & $0(0.0)$ & $1(100.0)$ & $0(0.0)$ & $0(0.0)$ & 1(100.0) & \\
\hline & $\begin{array}{c}\text { Rt. breast mass } \\
\text { excision }\end{array}$ & $0(0.0)$ & $0(0.0)$ & $0(0.0)$ & $0(0.0)$ & 1(100.0) & 1(100.0) & \\
\hline & Tonsillectomy & $0(0.0)$ & $1(50.0)$ & $1(50.0)$ & $0(0.0)$ & $0(0.0)$ & $2(100.0)$ & \\
\hline & Cholecystectomy & $0(0.0)$ & $0(0.0)$ & $0(0.0)$ & $4(57.1)$ & $3(42.9)$ & $7(100.0)$ & \\
\hline
\end{tabular}

$\mathrm{p}=0.000$, such that most of them did not have a surgical history, while a few of them $(\mathrm{n}=14)$ had surgical history of appendectomy Table (14).

pain was the most frequent presentation among the patients with different marital status, followed by epigastric pain Table (13). 


\begin{tabular}{|c||c|c|c|c||c|c||c||}
\hline \hline $\begin{array}{c}\text { Appendectomy } \\
\text { \& } \\
\text { Cholecystectomy }\end{array}$ & $0(0.0)$ & $0(0.0)$ & $0(0.0)$ & $0(0.0)$ & $1(100.0)$ & $1(100.0)$ \\
\hline \hline No PSH & $34(34.0)$ & $33(33.0)$ & $17(17.0)$ & $7(7.0)$ & $9(9.0)$ & $\begin{array}{c}100(100.0 \\
)\end{array}$ \\
\hline Total & $35(27.8)$ & $36(28.6)$ & $24(19.0)$ & $13(10.3)$ & $18(14.3)$ & $\begin{array}{c}126(100.0 \\
)\end{array}$ \\
\hline
\end{tabular}

Regarding the association between the patients' past surgical history (PSH) and their gender, the results of the present study showed that there was a significant association between the two variables at $\mathrm{p}=0.010$, such that most of them in both genders did not have a surgical history, while a few of them $(n=14)$ had surgical history of appendectomy Table (15).

Table (15): The association between the patients' past surgical history and their gender

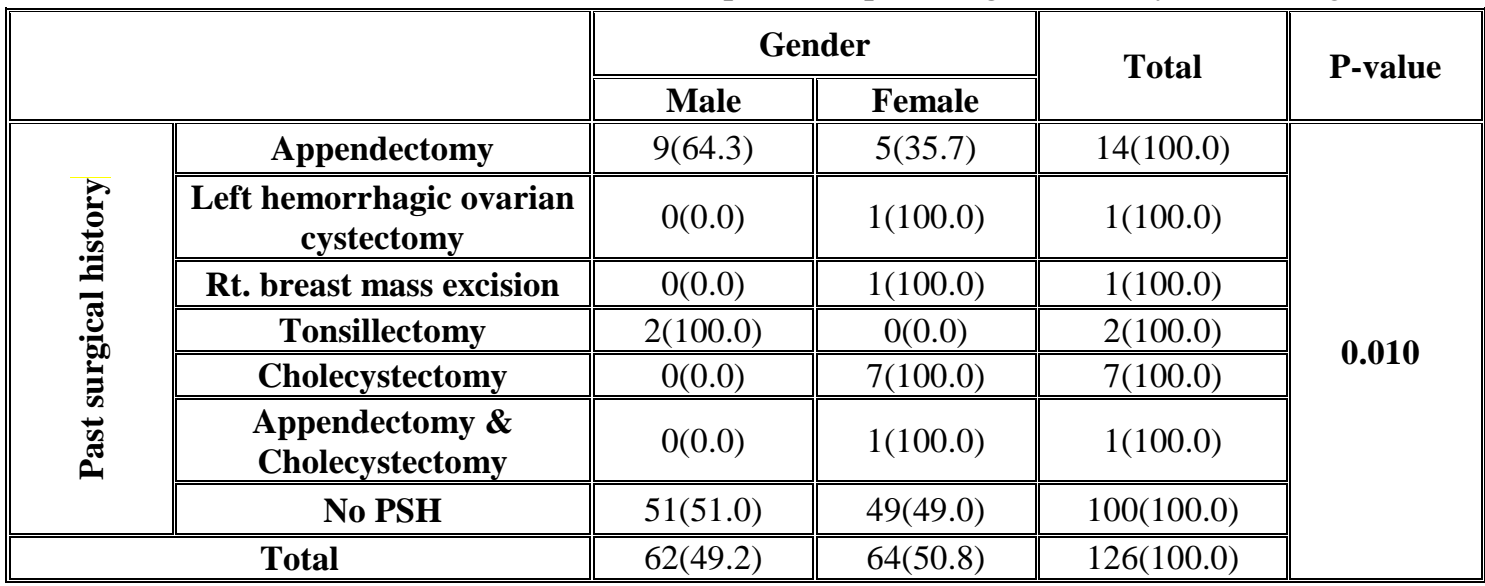

According to the results of the present study, there was a significant association between the readmitted patients' occupation and their past surgical history $(\mathrm{p}=0.003)$, such that most of them with different jobs $(\mathrm{n}=100)$ did not have a surgical history, while a few of them $(n=14)$ had surgical history of appendectomy Table (16).

Table (16): The association between the patients' past surgical history and their occupation

\begin{tabular}{|c|c|c|c|c|c|c|c|c|}
\hline & \multicolumn{5}{|c|}{ Occupation } & \multirow[b]{2}{*}{ Total } & \multirow[b]{2}{*}{$\begin{array}{c}\text { P- } \\
\text { value }\end{array}$} \\
\hline & & $\begin{array}{l}\text { Pupil \& } \\
\text { student }\end{array}$ & $\begin{array}{c}\text { Fixed } \\
\text { job }\end{array}$ & $\begin{array}{c}\text { Self- } \\
\text { employed }\end{array}$ & Unemployed & Housewife & & \\
\hline \multirow{4}{*}{ 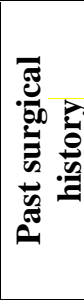 } & Appendectomy & $1(7.1)$ & $3(21.4)$ & $6(42.9)$ & $1(7.1)$ & $3(21.4)$ & 14(100.0) & \multirow{4}{*}{0.003} \\
\hline & $\begin{array}{c}\text { Left hemorrhagic } \\
\text { ovarian } \\
\text { cystectomy }\end{array}$ & $0(0.0)$ & $1(100.0)$ & $0(0.0)$ & $0(0.0)$ & $0(0.0)$ & $1(100.0)$ & \\
\hline & $\begin{array}{l}\text { Rt. breast mass } \\
\text { excision }\end{array}$ & $0(0.0)$ & $1(100.0)$ & $0(0.0)$ & $0(0.0)$ & $0(0.0)$ & $1(100.0)$ & \\
\hline & Tonsillectomy & $0(0.0)$ & $0(0.0)$ & $2(100.0)$ & $0(0.0)$ & $0(0.0)$ & $2(100.0)$ & \\
\hline
\end{tabular}




\begin{tabular}{|c||c||c||c||c|c||c||c||}
\hline & Cholecystectomy & $0(0.0)$ & $0(0.0)$ & $0(0.0)$ & $0(0.0)$ & $7(100.0)$ & $7(100.0)$ \\
\hline \hline $\begin{array}{c}\text { Appendectomy \& } \\
\text { Cholecystectomy }\end{array}$ & $0(0.0)$ & $0(0.0)$ & $0(0.0)$ & $0(0.0)$ & $1(100.0)$ & $1(100.0)$ \\
\hline \hline No PSH & $30(30.0)$ & $9(9.0)$ & $30(30.0)$ & $5(5.0)$ & $26(26.0)$ & $100(100.0)$ \\
\hline \hline Total & $31(24.6)$ & $14(11.1)$ & $38(30.2)$ & $6(4.8)$ & $37(29.4)$ & $126(100.0)$ \\
\hline
\end{tabular}

Regarding the association between the patients' past surgical history and their marital status, the results of the present study revealed that there was a significant relationship between these two variables at p-value of 0.005 , such that most of them with different marital statuses $(n=100)$ did not have a surgical history, followed by a few of them $(n=14)$ who had surgical history of appendectomy Table (17).

Table (17): The association between the patients' past surgical history and their marital status

\begin{tabular}{|c|c|c|c|c|c|c|c|}
\hline & & \multicolumn{4}{|c|}{ Marital status } & \multirow{2}{*}{ Total } & \multirow{2}{*}{ P-value } \\
\hline & & Single & Married & Widowed & Divorced & & \\
\hline \multirow{7}{*}{ 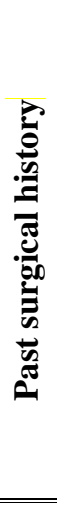 } & Appendectomy & $2(14.3)$ & $9(64.3)$ & $2(14.3)$ & $1(7.1)$ & $14(100.0)$ & \multirow{8}{*}{0.005} \\
\hline & $\begin{array}{c}\text { Left hemorrhagic } \\
\text { ovarian cystectomy }\end{array}$ & $0(0.0)$ & $1(100.0)$ & $0(0.0)$ & $0(0.0)$ & $1(100.0)$ & \\
\hline & $\begin{array}{l}\text { Rt. breast mass } \\
\text { excision }\end{array}$ & $0(0.0)$ & $0(0.0)$ & $1(100.0)$ & $0(0.0)$ & $1(100.0)$ & \\
\hline & Tonsillectomy & $1(50.0)$ & $1(50.0)$ & $0(0.0)$ & $0(0.0)$ & $2(100.0)$ & \\
\hline & Cholecystectomy & $0(0.0)$ & $5(71.4)$ & $2(28.6)$ & $0(0.0)$ & $7(100.0)$ & \\
\hline & $\begin{array}{c}\text { Appendectomy \& } \\
\text { Cholecystectomy }\end{array}$ & $0(0.0)$ & $0(0.0)$ & $1(100.0)$ & $0(0.0)$ & $1(100.0)$ & \\
\hline & No PSH & $44(44.0)$ & $49(49.0)$ & $6(6.0)$ & $1(1.0)$ & $100(100.0)$ & \\
\hline & Total & $47(37.3)$ & $65(51.6)$ & $12(9.5)$ & $2(1.6)$ & $126(100.0)$ & \\
\hline
\end{tabular}

\section{Discussion}

Different rates of readmission have been reported by different studies. For example, in their review, the collected data from 10 states of the United States, Jiang and Wier (2010) reported an approximate incidence rate of $10.7 \%$ for patients with Medicaid and $6.3 \%$ for those with private insurance 46. In another study in the USA, the readmission rate among trauma patients was 1.7[47].

Kociol et al. (2012) reported that readmission rates in the USA and non-US countries are respectively 10.5 and $7.7 \%$
[49]. A study carried out in Basra, Iraq indicated that readmission rate was $23.1 \%$ among children and adolescents with sickle cell disease [50]. In this a prospective study which lasted 3 months, the incidence rate of Hospital readmission was 38.6 cases out of $1000(3.86 \%)$. The difference in the rates reported by different studies can be related to the departments, types of disease, and settings that have been taken into consideration in different studies. Readmission after discharge from hospital is 
common and exerts remarkable economic burden on the patients and the health care systems[35]. Patterns of vulnerability to different conditions that were found to cause rehospitalization vary by time after the patient is discharge from the hospital. This finding suggests that recovery of various physiologic systems occurs at different rates and that post-discharge interventions to minimize vulnerability to specific conditions should be tailored to their underlying risks. However, the risk of rehospitalization is elevated in the immediate post-discharge period and declines over time. It is not known if the extent and timing of risk is different across readmission diagnoses, which suggests that recovery and vulnerability after discharge can differ by physiologic system. As could be seen from Table (1)\&(2), only $14.3 \%$ of the patients were older than 50 years old, while various studies have cited that the readmission rate of patients particularly increases with age 2, 46 ,also the rate of the female patients was $1.6 \%$ more than the male patients, but according to the reports of the study conducted by Hassan and Mohammad [47], no significant association could be found between gender and readmission. Based on the information presented $30.2 \%$ of the patients were selfemployed, and $29.4 \%$ of the women were housewives. However, according to the results of the study carried out by Madineh et al [48] study the patients' age, gender, marital status, occupation, education, ethnicity, access to insurance, smoking, or history of associated diseases did not have a significant effect on patient hospital readmission., by considering the effect of marital status on the patient's hospital readmission, it is clear that $51.6 \%$ of the patients were married, while $37.3 \%$ were single. However, as reported by Hassan and Mohammad [47], marital status could not be considered as a major effective factor in readmitting patients in hospital. The results of our study demonstrated that the most frequent presentation was right lower abdominal pain which was seen in half of patients and epigastric pain in in $21.4 \%$ of patients. On the other hand, the results that are presented in clearly prove this fact that one of the main symptoms that patients presented at the last admission was epigastric pain and dyspepsia with $33.3 \%$ followed by abdominal pain with $26.2 \%$. This results are similar to those of the studies carried out by Jesse et al [49] and Donze et al [35] who showed that abdominal and epigastric pain are the most common presentations among patients who may be readmitted to hospitals. In accordance with the information presented in Table (3), it could be seen that just one of the patients had a long hospitalization period of 36 days, and more than $50 \%$ of them stayed in hospital less than 5 days. However, some previous studies demonstrated that the duration of hospitalization could not have any specific effects on the potential of patient's hospital readmission [6, 48]. Ahmad et al [50] reported that history of medical associated diseases and surgical procedures may have a remarkable effect on the possibility of patient hospital readmission in 
the future, while the results of the present study concerning past surgical history revealed that $11.1 \%$ of the patients went with appendectomy, $5.6 \%$ went under surgical removal of the gallbladder and $79.4 \%$ of patients did not have any history of surgery Table (4).In the present study, some various lab investigations tests such as complete blood count (CBC), general urine examination (GUE), random blood sugar (RBS), liver function tests (LFTs), renal function test (RFT), amylase test, lipase test, lipid profile, blood urea nitrogen (BUN), electrocardiogram and high blood pressure, hemoglobin A1c or glycated hemoglobin test and C-reactive protein test were conducted on the patients to find more correlated risk factors to the patient's hospital readmission, however, these examinations have not been dealt with in previously conducted studies; therefore, no comparison can be done in this regard Table (5) and Table (8). In accordance with the aforementioned matters, the most common symptoms because of which the patients had been readmitted to the hospital were abdominal disorders, such that as could be seen in Table (6), abdominal ultrasonography and abdominal x-ray imaging studies were employed more than any other imaging tests. After diagnostic assessments were applied on the readmitted patients, it could be seen that suspected acute appendicitis and acute pancreatitis were the most common detected disorders that patients suffered from with the percentage of $37.3 \%$, $12.7 \%$ and $11.9 \%$, respectively. These findings cannot be compared to any other previously conducted studies, because they have not been studied in similar investigations. However, the results of this study showed that these disorders and problems could be considered as the most common reasons of the patients' hospital readmission. Approximately three-quarters of hospitalized patients are able to return to their home environment following discharge 18. For discharge home, patients with help from family or other caregivers, if available, should be able to obtain and self-administer medications, perform self-care activities, eat an appropriate diet or otherwise manage nutritional needs, follow up with designated providers. Here in the present study, the results in terms of the causes of the patients' discharge demonstrated that over $2 / 3$ of patients $(73 \%)$ were discharged because of pain relief and delicate management, $14.3 \%$ of them were discharged upon their responsibility by their own demand and other patients discharged because of pain mitigate and also due to their improved health condition Table (9).Hansen et al [1] have classified various kinds of patient's interventions to pre-discharge interventions, post-discharge interventions and bridging interventions. However, they pointed out that various interventions in each step are essential for reduction of patient's hospital readmission rate. In our study, the information that is presented in Table (10) showed that $87.3 \%$ of the patients underwent conservative management, $7.1 \%$ of them were prepared for their appendix and two of them had been suspected. Therefore, it could 
be seen that approximately a broad range of patients in this study had experienced previous intervention that is a very important factor in reduction of patient's hospital readmission.Analyzing the relationship between the readmitted patients' age and the observed presentations demonstrated that these two variables were significantly correlated ( $p=0.002)$. This finding is in good agreement with the results of the study conducted by Gheorghiade et al. (2013) who studied problems and perspectives in heart failure patients who were re-hospitalized. In their study, the reported a significant association between the readmitted patients' and their type of disease and clinical symptoms [51]. The relationship between the type of clinical presentations and the readmitted patients' age has hardly been studied; however, most studies have introduced age as a risk factor for being rehospitalization [52-54]. The results of the present study also indicated that the readmitted patients' occupation and their clinical representations were significantly correlated $(\mathrm{p}=0.01)$. This finding cannot be compared to those of any previous studies, because no study has focused on the association between types of clinical representations and the readmitted patients' occupation. However, some studies have indicated that demographic characteristics such as age and gender can have an effect on readmission rates $[53,55,56]$.According to the results of the present study, the readmitted patients' marital status had a significant effect on the type of their clinical presentation at readmission $(\mathrm{p}=0.007)$. The correlation between the types of clinical presentation and marital status has never been studied before; therefore, this finding cannot be compared to any previously reported ones. However, different studies have indicated that marital status can significantly affect the rates of readmission, such that married patients had fewer events of readmission [57]. However, Adogwa et al. (2017) observed no significant difference between patients with different marital statuses in terms of the rate of their hospital readmission $(p=0.99)$ [58].The results of the present study revealed that there was a significant relationship between the readmitted patients' age and their past surgical history $(p=0.000)$ although most of the patients' had no past surgical history. The most frequently observed past surgery was appendectomy. In line with this finding, Moghadamyeghaneh et al. (2016) reported that $3.7 \%$ of the patients who had undergone appendectomy experienced hospital readmission59.Also, the results of the current investigation showed that the readmitted patients' gender and their past surgical history were significantly correlated $(\mathrm{p}=0.10)$ although most of them did not have a past surgical history. As observed in Table (15) above, 14 patients (of both genders) who had undergone appendectomy were rehospitalized. As mentioned above, patients' with a history of appendectomy have some odds of being readmitted [59]. However, no previously conducted study have focused on the exact relationship between the re- 
hospitalized patients' past surgical history and their gender; therefore, no comparison can be done in this regard.The results also indicated a significant relationship between the patients' occupation and their past surgical history at a p-value of 0.003 . However, this relationship has not been focused on in any previously conducted studies; therefore, it cannot be compared to any previously reported ones. Finally the results revealed a significant correlation between the patients' marital status and their past surgical history $(\mathrm{p}=0.005)$. This finding cannot be compared to any previous ones, either, because similar studies have not focused on the relationship between these two variables.

\section{Conclusions}

-The incidence rate of hospital readmission in our prospective study is 38.6 cases out of $1000(3.86 \%)$.

- Readmission is high in the immediate postdischarge period and decreases over time.

- The rate of hospital readmission was lower among the patients $>50$ years.

- Female patients accounted for a larger population of readmitted patients.

- No significant association was found between gender and readmission.

- The most frequent symptoms and presentations observed at readmission were epigastric and abdominal pains.

- Associated medical diseases and surgical procedures were found to have outstanding effect on readmission.

- The most common detected disorders among the readmitted patients were suspected acute appendicitis and acute pancreatitis.

- The type of medical intervention has a remarkable effect on the rate of readmission.

- There was a significant relationship between the observed clinical presentations and the readmitted patients' age, occupation, and marital status.

- There was a significant relationship between the readmitted patients' past surgical history and their age, gender, occupation, and marital status.

\section{Recommendations}

(1) Based on the correlation between the type of medical intervention and readmission rate, future studies should focus specifically on this probable correlation and pay closer attention to those patients who receive interventions that might raise hospital readmission.

(2) Since readmission rate is higher during the first days after discharge and lower over time, special care should be provided to patients who are most suspected to be readmitted.

(3) Patients with right lower abdominal pain, epigastric pain, history of internal problems and surgical procedures, acute appendicitis, and acute pancreatitis who are more prone to be readmitted need to be paid closer attention.

(4) Since patients' age, occupation, and marital status are correlated with the type of clinical presentations among readmitted patients, these factors need to be taken in good consideration while admitting and readmitting patients. 
(5) Given the significant relationship between the readmitted patients' past surgical history and their age, gender, occupation, and marital status, these factors should be taken in close account while the patients are admitted or readmitted.

\section{References}

[1]Hansen LO, Young RS, Hinami K, et al. Interventions to reduce 30-day rehospitalization: a systematic review. Ann Intern Med 2011; 155:520.

[2]Jencks SF, Williams MV, Coleman EA. Rehospitalizations among patients in the Medicare fee-for-service program. N Engl J Med. 2009;360(14):1418-28.

[3]Gerhardt G, Yemane A, Apostle K, Oelschlaeger A, Rollins E, Brennan N. Evaluating whether changes in utilization of hospital outpatient services contributed to lower Medicare readmission rate. Medicare Medicaid Res Rev. 2014;4(1).

[4]Frei-Jones MJ, Field JJ, DeBaun MR. Risk factors for hospital readmission within 30 days: a new quality measure for children with sickle cell disease. Pediatrics Blood Cancer. 2009;52(4):481-5.

[5] Morris DS, Rohrbach J, Rogers M, Thanka Sundaram LM, Sonnad S, Pascual J, et al. The surgical revolving door: risk factors for hospital readmission. J Surg Res. 2011;170(2):297-301.

[6]Owoicho A, Aladine A. E, Victoria D. V, et al. Effect of Social Support and Marital Status on Perceived Surgical Effectiveness and 30-Day Hospital Readmission. Global Spine Journal 2017, Vol. 7(8) 774-779.
[7] Elizabeth M. G, Blake M, Rouba Y, et al. An analysis of diagnoses that drive readmission: What can we learn from the hospitals in Southern New England with the highest and lowest readmission performance? R I Med J. 2017 Aug 1; 100(8): 23-28. [8] Dharmarajan K, Hsieh AF, Kulkarni VT, et al. Trajectories of risk after hospitalization for heart failure, acute myocardial infarction, or pneumonia: retrospective cohort study. BMJ (Clinical Research Ed.). 2015;350: h411.

[9] Ash AS, Fienberg SE, Louis TA, et al. COPSS-CMS White Paper Committee. 2012. Statistical issues in assessing hospital performance.

[10]Sheingold, Steven H.; Zuckerman, Rachael; Shartzer, Adele (2016-01-01). "Understanding Medicare Hospital Readmission Rates and Differing Penalties between Safety-Net and Other Hospitals". Health Affairs (Project Hope). 35 (1): 124131.

[11]Schmidt S, Sheikzadeh S, Beil B, et al. Acceptance of telemonitoring to enhance medication compliance in patients with chronic heart failure. Telemed J E Health. 2008;14:426-33.

[12] Kuo YF, Goodwin JS. Association of hospitalist care with medical utilization after discharge: evidence of cost shift from a cohort study. Ann Intern Med 2011; 155:152. [13] Harb N, Foster JM, Dobler CC. Patientperceived treatment burden of chronic obstructive pulmonary disease. Int J Chron Obstruct Pulmon Dis. 2017;12:1641-1652. [14]Dobler CC. Defragmenting health care 
for patients with chronic obstructive pulmonary disease. AMJ. 2016;9(6):185188.

[15]Audit Scotland. Managing Long-term Conditions. 2007. Available from: http://www.auditscotland.gov.uk/docs/health/2007/nr-070816managing-long-term.pdf.

Accessed

November 2, 2017.

[16]Harries $\mathrm{TH}$, Thornton $\mathrm{H}$, Crichton S, Schofield P, Gilkes A, White PT. Hospital readmissions for COPD: a retrospective longitudinal study. NPJ Prim Care Respir Med. 2017; 27:31.

[17]Feemster LC, $\mathrm{Au}$ DH. Penalizing hospitals for chronic obstructive pulmonary disease readmissions. Am J Respir Crit Care Med. 2014;189(6):634-639.

[18]Bureau of Health Information [webpage on the Internet]. The Insights Series Exploring Clinical Variation in Readmission, Return to Acute Care Following Discharge from Hospital, Eight Clinical Conditions, NSW, July 2012 - June 2015. Sydney, NSW: BHI; 2017.

[19]Shams I, Ajorlou S, Yang K. A predictive analytics approach to reducing 30day avoidable readmissions among patients with heart failure, acute myocardial infarction, pneumonia, or COPD. Health Care Manage Sci. 2015;18(1):19-34.

[20]Sjoding MW, Cooke CR. Readmission penalties for chronic obstructive pulmonary disease will further stress hospitals caring for vulnerable patient populations. Am J Respir Crit Care Med. 2014;190(9):1072-1074.
[21]Woodside, M. (1953-01-01). "A followup of psychiatric patients; one year's survey of patients discharged from the York Clinic". Guy's Hospital Reports. 102 (1): 70-75. ISSN 0017-5889. PMID 13034097.

[22]Wiseman, Jason T.; Guzman, Amanda M.; Fernandes-Taylor, Sara; Engelbert, Travis L.; Saunders, R. Scott; Kent, K. Craig (2014-09-01). "General and vascular surgery readmissions: a systematic review". Journal of the American College of Surgeons. 219 (3): 552-569.e2.

[23] Van Walraven C, Bennett C, Jennings A, Austin PC, Forster AJ. Proportion of hospital readmissions deemed avoidable: a systematic review.CMAJ. 2011a: cmaj. 101860.

[24]Axon RN, Williams MV. Hospital readmission as an accountability measure. JAMA. 2011;305(5):504-5.

[25]Kripalani S, Theobald CN, Anctil B, Vasilevskis EE. Reducing hospital readmission rates: current strategies and future directions. Annu Rev Med. 2014; 65:471-85.

[26] Dunlay SM, Redfield MM, Weston SA, et al. Hospitalizations after heart failure diagnosis a community perspective. J Am Coll Cardiol. 2009;54: 1695-702.

[27] Dharmarajan K, Hsieh AF, Lin Z, et al. Diagnoses and timing of 30-day readmissions after hospitalization for heart failure, acute myocardial infarction, or pneumonia. JAMA. 2013;309: 355-63.

[28] Harlan M. K, Angela H, Rachel P. D, et al. Trajectories of Risk for Specific Readmission Diagnoses after Hospitalization 
for Heart Failure, Acute Myocardial Infarction, or Pneumonia. PLoS One. 2016; 11(10): e0160492.

[29] Krumholz HM. Medicine in the era of outcomes measurement. Circulation: Cardiovasc Qual Outcomes. 2009; 2:141143.

[30] Phillips CO, Wright SM, Kern DE, et al. Comprehensive discharge planning with post discharge support for older patients with congestive heart failure: a meta-analysis. JAMA. 2004;291: 1358-1367.

[31]Hospital Compare. Department of Health and Human Services. http:// www.hospitalcompare.hhs.gov. Accessed 24 June 2009.

[32] Waljee J, McGlinn EP, Sears ED, Chung KC. Patient expectations and patient-reported outcomes in surgery: a systematic review. Surgery. 2014;155:799-808.

[33]Rosenberger PH, Jowl P, Ickovics J. Psychosocial factors and surgical outcomes: an evidence-based literature review.JAmAcad Orthop. Surg. 2006;14:397405.

[34]Gatchel RJ, Mayer TG,KidnerCL,McGearyDD.Are gender, marital status or parenthood risk factors for outcome of treatment for chronic disabling spinal disorders?J Occup Rehabil. 2005; 15:191-201.

[35] Donze J, Aujesky A, Williams D, et al. Potentially avoidable 30-day readmissions in medical patients. Derivation and validation of a predictive model. JAMA Intern Med. 2013;173(8):632-8.
[36]Reducing Readmissions. NHS Innovation \& Improvement. Available from: http://webarchive.nationalarchives.gov.uk/20 121108093302/http://www.

institute.nhs.uk/quality_and_service_improve ment_tools/quality-andservice_improvement_tools/discharge_planni ng.html Accessed: 13th Oct 2017.

[37]Krumholz HM. Post-hospital syndromean acquired, Transient Condition of Generalized Risk. N Engl J Med. 2013;368(2):100-2.

[38]Van Walraven JA, Taljaard M, et al. Incidence of potentially avoidable urgent readmissions and their relation to all-cause urgent readmissions. CMAJ. 2011b;183(14): E1067-72.

[39] Cotter PE, Bhalla VK, Wallis SJ, Biram RW. Predicting readmissions: poor performance of the LACE index in an older UK population. Age Ageing. 2012;41:784-9. [40]Billings J, Blunt I, Steventon A, Georghiou LG, Bardsley M. Development of a predictive model to identify inpatients at risk of re-admission within 30 days of discharge (PARR-30). BMJ Open. 2012;2: e001667.

[41]Ottenbacher KJ, Karmarkar A, Graham JE, et al. Thirty-day hospital readmission following discharge from post-acute rehabilitation in fee-for-service Medicare patients. JAMA. 2014;311(6):604-14.

[42]Fisher SR, Graham JE, Krishnan S, Ottenbacher KJ. Predictors of 30-day readmission following inpatient rehabilitation for patients at high risk for hospital readmission. Phys Ther. 2016;96(1):62-70. 
[43]Ngui NK, Hitos K, Ctercteko G. Preoperative factors prolonging the length of stay in elective colorectal surgery.ANZ J Surg. 2011;81:624-628.

[44] Marek Z, Andreas L, Christian S, et al. Assessment and pathophysiology of pain in cardiac surgery. J Pain Res. 2018; 11: 15991611 .

[45]Zhe Z, Dmitry T. Expected social support and recovery of functional status after heart surgery. Disable Rehabil. 2019 Jan 7:1-6.

[46] Jiang HJ, Wier LM. All-cause hospital readmissions among non-elderly Medicaid patients, 2007. 2010.

[47]Ladha KS, Young JH, Ng DK, Efron DT, Haider AH. Factors affecting the likelihood of presentation to the emergency department of trauma patients after discharge. Ann Emerg Med. 2011;58(5):431-7. doi: 10.1016/j.annemergmed.2011.04.021.

[48] Kociol RD et al. International variation in and factors associated with hospital readmission after myocardial infarction. JAMA 2012 Jan 4; 307:66.

[49] Salman ZA, Hassan MK. Hospitalization events among children and adolescents with sickle cell disease in Basra, Iraq. Anemia 2015;2015:195469.

[50] Moloney ED, Bennett K, Silke B. Patient and disease profile of emergency medical readmissions to an Irish teaching hospital. Postgrad Med J. 2004 Aug;80(946):470-4.

[51] Hassan G, Mohammad H K M. Hospital Readmission Through the Emergency
Department. Trauma Mon. 2016 May; 21(2): e35139.

[52]Madineh D, Nasrin E, Shahram B, et al. The Effects of Group Education with the Teach-Back Method on Hospital Readmission Rates of Heart Failure Patients. Jundishapur Journal of Chronic Disease Care: January 01, 2016, 5 (1); e30377.

[53] Jesse J. B, Theodore C. C, James P. K, et al. Inpatient Readmissions and Emergency Department Visits within 30 Days of a Hospital Admission. West J Emerg Med. 2015;16(7):1025-1029.

[54] Ahmad K S, Kamran B L, Reza T, et al. Evaluation of 30-Day Unplanned Hospital Readmission in a Large Teaching Hospital in Shiraz, Iran. Shiraz E-Medical Journal: April 01, 2017, 18 (4); e39745.

[55]Gheorghiade M, Vaduganathan M, Fonarow GC, Bonow RO. Rehospitalization for heart failure: problems and perspectives. J Am Coll Cardiol. 2013;61(4): 391-403.

[56] Yandrapalli S, Aronow WS, Frishman WH. Readmissions in adult patients following hospitalization for influenza: a nationwide cohort study. Ann Transl Med. 2018;6(16):318.

doi:10.21037/atm.2018.07.18

[57]Aljishi M, Parekh K. Risk factors for general medicine readmissions and association with mortality. $\mathrm{N} Z$ Med J. 2014;127(1394):42e50.

[58] Prescott HC, Sjoding MW, Iwashyna TJ. Diagnoses of early and late readmissions after hospitalization for pneumonia. A systematic review. Ann Am Thoracic Soc. 
2014;11(7):1091-1100.

doi:10.1513/AnnalsATS.201404-142OC

[59] Herrin J, St Andre J, Kenward K, Joshi

MS, Audet AM, Hines SC. Community

factors and hospital readmission rates. Health

Serv Res. 2014;50(1):20-39.

doi:10.1111/1475-6773.12177.

[60]Barnett ML, Hsu J, McWilliams JM.

Patient Characteristics and Differences in

Hospital Readmission Rates. JAMA Intern

Med. 2015;175(11):1803-1812.

doi:10.1001/jamainternmed.2015.4660.

[61]Hu J, Gonsahn MD, Nerenz

DR.Socioeconomic status and readmissions:

evidence from an urban teaching hospital.Health Aff (Millwood). 2014; 33:778-785. doi: 10.1377/hlthaff.2013.0816.

[62] Adogwa O, Elsamadicy AA, Vuong VD, et al. Effect of Social Support and Marital Status on Perceived Surgical Effectiveness and 30-Day Hospital Readmission. Global Spine J. 2017;7(8):774-779. doi:10.1177/2192568217696696 [63]Moghadamyeghaneh Z, Hwang G, Hanna MH, Carmichael JC, Mills S, Pigazzi A, Stamos MJ. Unplanned readmission after appendectomy. Am J Surg. 2016;212(3):493500. 